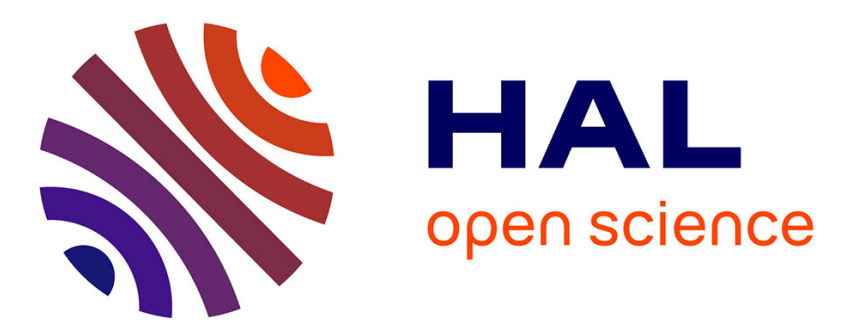

\title{
Using A Quadrotor As Wind Sensor: Time-Varying Parameter Estimation Algorithms
}

Gabriele Perozzi, Denis Efimov, Jean-Marc Biannic, Laurent Planckaert

\section{To cite this version:}

Gabriele Perozzi, Denis Efimov, Jean-Marc Biannic, Laurent Planckaert. Using A Quadrotor As Wind Sensor: Time-Varying Parameter Estimation Algorithms. International Journal of Control, inPress, 95 (1), pp.126-137. 10.1080/00207179.2020.1780324 . hal-02784701

\section{HAL Id: hal-02784701 \\ https://inria.hal.science/hal-02784701}

Submitted on 4 Jun 2020

HAL is a multi-disciplinary open access archive for the deposit and dissemination of scientific research documents, whether they are published or not. The documents may come from teaching and research institutions in France or abroad, or from public or private research centers.
L'archive ouverte pluridisciplinaire HAL, est destinée au dépôt et à la diffusion de documents scientifiques de niveau recherche, publiés ou non, émanant des établissements d'enseignement et de recherche français ou étrangers, des laboratoires publics ou privés. 


\title{
CONTRIBUTION PAPER
}

\section{Using A Quadrotor As Wind Sensor: Time-Varying Parameter Estimation Algorithms}

\author{
Gabriele Perozzi ${ }^{\mathrm{a}, \mathrm{b}, *}$, Denis Efimov ${ }^{\mathrm{c}}$, Jean-Marc Biannic ${ }^{\mathrm{d}}$ and Laurent Planckaert ${ }^{\mathrm{a}, \mathrm{b}}$ \\ aONERA - The French Aerospace Lab, DAAA, Lille F-59014, France. \\ ${ }^{\mathrm{b}}$ LMFL - Laboratoire de Mécanique des Fluides de Lille - Kampéde Fériet, Lille F-59000, France. \\ ' Inria, Universitéde Lille, CNRS, UMR 9189 - CRISTAL, Lille F-59000, France. \\ ${ }^{\mathrm{d}}$ ONERA - The French Aerospace Lab, DTIS, Toulouse F-31055, France.
}

\author{
ARTICLE HISTORY \\ Compiled May 29, 2020
}

\begin{abstract}
The objective of this paper is to develop an algorithm for the estimation of time-varying wind parameters by taking into account a detailed quadrotor model. The design objectives include the time convergence optimization, robustness to measurement noises, and a guaranteed convergence of the estimates to the true values under mild applicability conditions. It is supposed that the estimation algorithm can use IMU (accelerometers, gyroscopes) sensors augmented with an earth reference tracking system and rotor rotational velocity sensors. To this end, three time-varying parameter estimation algorithms are introduced, compared and finally merged to estimate the varying wind velocity in on-board quadrotor systems. Final numerical experiments, using a nonlinear quadrotor simulator, are used to validate the proposed approaches.
\end{abstract}

\section{KEYWORDS}

Quadrotor, wind velocity estimation, time-varying parameter estimation.

\section{Introduction}

Unmanned Aerial Vehicle (UAV) quadrotors are often required to move in unfamiliar environments in terms of geography and wind conditions. The effect of the wind on small quadrotor vehicles can lead to dangerous situations when they operate in close proximity to physical obstacles or other aerial vehicles. Thus, to allow UAVs to operate in urban environments, inside turbulent air flow patterns for which accurate prediction is not available a priori, the on-board estimation of wind speed becomes essential to adapt control algorithms. The problem is that the airspeed sensors, such as aeroclinometers and Pitot tubes, are not easily usable with rotary wing vehicles because the inflow of the rotors interferes with the atmospheric flow. Further, adding on-board wind sensors limits other additional valuable payload. Another approach to estimate the wind is related with an estimation scheme (or an intelligent virtual sensor), which has to be designed based on an adequate drone model and measurements available on quadrotors in inertial tracking position system. According to the aerodynamic science, a nonlinear dependence of the UAV behavior on the wind speeds comes out, while the disturbances (external forces and moments) enter linearly in the drone equations. Hence, the problems of estimation of wind velocities and disturbances can be posed assuming them constant or slowly varying (analysis of time-varying systems is much harder and it requires more effort, see, for

\footnotetext{
* corresponding author: Gabriele Perozzi. Email: gabrieleperozzi@ hotmail.com
} 
example, Zhou \& Egorov (2016), Zhou (2016),Ning, He, Wu, \& She (2014), and Peng \& Zhang (2010)). The following articles propose wind and disturbance estimations without the use of additional airspeed sensors.

Xing, Qu, \& Zhang (2017) estimated the shear wind vector at low altitude using IMU and GNSS module. Pappu, Liu, Horn, \& Cooper (2017) used a Kalman filter based identification technique for estimating wind gusts. The method described by Lie \& Gebre-Egziabher (2013) relied on measurements from GPS, an IMU, and a low-fidelity model of the aircraft's dynamics, which are fused using two cascaded extended Kalman filters. Pendleton \& Zhang (2017) estimated the wind using the drone model in hover flight. Demitrit, Verling, Stastny, Melzer, \& Siegwart (2017) addressed the problem of onboard wind estimation for a hovering vertical takeoff and landing tailsitter UAV. Gonzalez-Rocha, Woolsey, Sultan, de Wekker, \& Rose (2017) used the kinematic particle model and dynamic particle model with identified motion model parameters. Tomić \& Haddadin (2014) presented a model-based method for external wrench estimation in flying robots based on proprioceptive sensors and the robot's dynamics model. Tomić, Schmid, Lutz, Mathers, \& Haddadin (2016) described two complementary methods using the estimation of the external wrench and the estimation of the propeller aerodynamic power. Yüksel, Secchi, Bülthoff, \& Franchi (2014) presented Lyapunov method for external forces and moments in flying robots. Martínez-Vásquez et al. (2015) implemented a linear observer with integral action for estimating the disturbances due to a wind in hover flight mode. Qu, Xing, \& Zhang (2016) used a decomposition of the hovering state equations to estimate the wind, and Qu, Xing, Zhang, \& Yu (2017) extended the work using both IMU and a smoothing filter to reduce the effect of sensor noise. Witte et al. (2016) used a method based on on-board moving velocity sensors data such as five-hole and hot-wire probes. Benallegue, Mokhtari, \& Fridman (2008) built the high-order sliding mode observer as an estimator of the effect of the external disturbances in quadrotors such as wind and noise, using a differential global positioning system, a GPS, and a sonar altimeter. Santosuosso, Benzemrane, \& Damm (2011) developed a nonlinear strategy to estimate the translational velocity vector based on acceleration, angles and angular speeds measurements. Boiko \& Chehadeh (2018) used a sliding mode differentiator to estimate the quadrotor velocity with fusion of the position sensor from the motion capture optical system and accelerometer. Ali, Shah, Samar, \& Waseem (2016) used the high order sliding mode differentiator to estimate the wind velocity for a fixed-wing $\mathrm{UAV}$, using the rate of change of heading of the vehicle. Other works that use IMU and motion tracking system were carried out by Neumann \& Bartholmai (2015); Rhudy et al. (2015); Sikkel, Croon, Wagter, \& Chu (2016); Song et al. (2016); Waslander \& Wang (2009); Xiang et al. (2016). All the mentioned papers performed the estimation using physical models with different assumptions based on the available sensors.

In the present work, the design objectives include the time convergence optimization and robustness to measurement noises, and a guaranteed convergence of the estimates to the true values under mild applicability conditions. It is supposed that the estimation algorithm can use IMU (accelerometers, gyroscopes) sensors augmented with an earth reference tracking system and rotor rotational velocity sensors.

The contribution of this paper is to develop an on-board algorithm for the estimation of time-varying wind parameters by taking into account a detailed physical model given in Planckaert \& Coton (2015). The proposed approach has several notable differences with respect to conventional solutions used in practice, in particular with the Kalman Filter (KF) and the Extended Kalman Filter (EKF) based strategies. KFs and EKFs are widely used in the aerospace engineering, despite they have plenty of shortages, especially in the context of flying robots having a limited computational capacity. Indeed, in order to ensure optimality of the estimation, KF uses additional computational loops for gain adjustment, but the optimality of KF is usually lost if there is any imperfection in the model, which is always the case in 
practical applications. Moreover, the stability proofs for EKF are obtained under rather severe assumptions (see for instance, Karvonen (2014)). To avoid these issues, alternative algorithms, specifically tailored to our quadrotor model are proposed in this paper. The advantages of the proposed algorithms are that the stability conditions of these solutions are provided, and that these algorithms have a lower computational complexity than the KFs. To this end, three time-varying parameter estimation algorithms are introduced, compared and finally merged to estimate the varying wind velocity in on-board quadrotor systems.

The paper outline is as follows. In Section 2 the quadrotor model is presented. In Section 3 a study of the dynamics is carried out. Section 4 presents the wind estimation algorithms. Section 5 validates the proposed estimation algorithm with advanced simulations. Final remarks and discussion conclude the paper in Section 6. The stability concept definitions are summarized in Appendix.

\section{Flight dynamics}

The presented work is based on the commercial available Parrot Ar. Drone 2.0. Our model is based on standard notion where $\phi, \theta, \psi$ respectively denote the roll, pitch, yaw angles around $x, y, z$ axes, with $z$ positive direction is toward down. The translation dynamics of the drone in the body frame yield

$$
m\left[\begin{array}{c}
\dot{u} \\
\dot{v} \\
\dot{w}
\end{array}\right]+m\left[\begin{array}{c}
p \\
q \\
r
\end{array}\right] \times\left[\begin{array}{c}
u \\
v \\
w
\end{array}\right]=\left[\begin{array}{c}
F_{\text {Xaero }} \\
F_{\text {Yaero }} \\
F_{\text {Zaero }}
\end{array}\right]+m \mathbf{R}^{T}\left[\begin{array}{c}
0 \\
0 \\
g
\end{array}\right],
$$

where $m$ is the mass of the vehicle, $(u, v, w)$ are the linear velocities expressed in body frame, $(p, q, r)$ are the angular velocities in body frame, $\left(F_{\text {Xaero }}, F_{\text {Yaero }}, F_{\text {Zaero }}\right)$ are the external aerodynamic forces in body frame, $\mathbf{R}$ is the rotational matrix. The rotational dynamics of the drone with respect to Earth frame are

$$
\mathbf{I}\left[\begin{array}{c}
\dot{p} \\
\dot{q} \\
\dot{r}
\end{array}\right]=-\left[\begin{array}{c}
p \\
q \\
r
\end{array}\right] \times \mathbf{I}\left[\begin{array}{c}
p \\
q \\
r
\end{array}\right]+\left[\begin{array}{c}
L_{\text {aero }} \\
M_{\text {aero }} \\
N_{\text {aero }}
\end{array}\right]+\left[\begin{array}{c}
-J_{\text {rot }} q \Omega_{r} \\
J_{\text {rot }} p \Omega_{r} \\
-J_{\text {rot }} \dot{\Omega}_{r}
\end{array}\right]
$$

where $\left(L_{\text {aero }}, M_{\text {aero }}, N_{\text {aero }}\right)$ are the external aerodynamic moments in the body frame, $\Omega_{r}$ is the propeller angular rate, $J_{r o t}$ is the propeller inertia, $\mathbf{I}$ is the quadrotor inertia square matrix defined as $\mathbf{I}=\operatorname{diag}\left\{I_{x x}, I_{y y}, I_{z z}\right\}$ where $\left(I_{x x}, I_{y y}, I_{z z}\right)$ are the inertia of the drone around $(x, y, z)$ axes respectively and the other components out of the main diagonal of this matrix are not considered since they are very small. These three inertia values are linked together by the approximation $I_{z z} \approx I_{x x}+I_{y y}$. According to the identification work of Planckaert \& Coton (2015) performed at moderate speeds in forward, lateral, and vertical directions of $\pm 5, \pm 5 \pm 1 \mathrm{~m} / \mathrm{s}$ respectively, the gyroscopic effects, caused by the varying in orientation of the propeller plane, and the inertial counter torques, caused by the varying in propeller rotation speed, can be neglected since they are rather small. The relations between angular velocities and Euler angles are considered avoiding the singularity at $\theta=\frac{\pi}{2}$, which is a reasonable assumption in our case since the topic of this paper is not to achieve extreme maneuvers. The aerodynamic forces $\left(F_{\text {Xaero }}, F_{\text {Yaero }}, F_{\text {Zaero }}\right)$, moments $\left(L_{\text {aero }}, M_{\text {aero }}, N_{\text {aero }}\right)$, and related coefficients are presented below using the blade element momentum theory in helicopters, well explained by Bramwell, Balmford, \& Done (2001); Johnson (2012); Leishman (2006). Aerodynamic forces and moments for each rotor, where subscript $j$ indicates the $j^{\text {th }}$ rotor, are 
derived as

$$
\begin{aligned}
F_{X j} & =-\rho A R^{2} \frac{u_{j}-u_{w}}{\sqrt{\left(u_{j}-u_{w}\right)^{2}+\left(v_{j}-v_{w}\right)^{2}}} C_{H j} \omega_{j}^{2}, \\
F_{Y j} & =-\rho A R^{2} \frac{v_{j}-v_{w}}{\sqrt{\left(u_{j}-u_{w}\right)^{2}+\left(v_{j}-v_{w}\right)^{2}}} C_{H j} \omega_{j}^{2}, \\
F_{Z_{j}} & =-\rho A R^{2} C_{T_{j}} \omega_{j}^{2}, \\
L_{j} & =-\operatorname{sign} \omega_{j} \rho A R^{3} \frac{u_{j}-u_{w}}{\sqrt{\left(u_{j}-u_{w}\right)^{2}+\left(v_{j}-v_{w}\right)^{2}}} C_{R m_{j}} \omega_{j}^{2}, \\
M_{j} & =-\operatorname{sign} \omega_{j} \rho A R^{3} \frac{v_{j}-v_{w}}{\sqrt{\left(u_{j}-u_{w}\right)^{2}+\left(v_{j}-v_{w}\right)^{2}}} C_{R m_{j}} \omega_{j}^{2}, \\
N_{j} & =-\operatorname{sign} \omega_{j} \rho A R^{3} C_{Q_{j}} \omega_{j}^{2},
\end{aligned}
$$

where sign $\omega_{j}$ is the direction of the rotor angular velocities which can be positive or negative according to the rotation convention, $\rho$ is the air density, $A$ is the rotor area, $R$ is the rotor radius, $\left(u_{w}, v_{w}, w_{w}\right)$ are the wind velocities with respect to the Earth and in body frame respectively in $(x, y, z)$ directions, $C_{H j}$ is the hub force coefficient, $C_{T j}$ is the rotor thrust coefficient, $\omega_{j}$ is the rotor angular speed, $C_{Q_{j}}$ is the rotor drag moment coefficient, $C_{R m j}$ is the rotor rolling moment coefficient. The translational rotor velocities in body frame $\left(u_{j}, v_{j}, w_{j}\right)$ are computed as a function of the state in body frame $\left(u_{j}, v_{j}, w_{j}\right)^{T}=(p, q, r)^{T} \times\left(l c_{j}, l s_{j}, h\right)^{T}+(u, v, w)^{T}$ with $\mathrm{c}_{j}=\cos \left(\frac{\pi}{2}(j-1)+\varepsilon\right), \quad \mathrm{s}_{j}=\sin \left(\frac{\pi}{2}(j-1)+\varepsilon\right)$, where $h$ is the distance between the rotors' plane and the center of gravity of the vehicle, $l$ is the arm length, and $\varepsilon=\frac{\pi}{4}$ is the angle between the forward axis direction $x$ and the quadrotor arm considered positive in clockwise direction. Thus, for vectors $\mathrm{c}_{j}$ and $\mathrm{s}_{j}$ we have cosines and sines of the angles $\left[\frac{\pi}{4}, \frac{3}{4} \pi, \frac{5}{4} \pi, \frac{7}{4} \pi\right]$. Total aerodynamic forces are

$$
F_{\text {Xaero }}=\sum_{j=1}^{4} F_{X j}, \quad F_{\text {Yaero }}=\sum_{j=1}^{4} F_{Y j}, \quad F_{\text {Zaero }}=\sum_{j=1}^{4} F_{Z_{j}} .
$$

Total aerodynamic moments are

$$
\begin{aligned}
L_{\text {aero }} & =\sum_{j=1}^{4}\left(L_{j}+F_{Z j} l \mathrm{~s}_{j}-h F_{Y j}\right), \\
M_{\text {aero }} & =\sum_{j=1}^{4}\left(M_{j}-F_{Z j} l \mathrm{c}_{j}+h F_{X j}\right), \\
N_{\text {aero }} & =\sum_{j=1}^{4}\left(N_{j}+F_{Y j} l \mathrm{c}_{j}-F_{X j} l \mathrm{~s}_{j}\right) .
\end{aligned}
$$

The aerodynamic coefficients $\left(C_{T}\right.$ coefficient of the thrust, $C_{R m}$ coefficient of the rolling moment, $C_{H}$ coefficient of the hub force, $C_{Q}$ coefficient of the drag moment, $\mu$ advance ratio, $\lambda$ inflow ratio) are computed as follows using blade element momentum theory and under the 
hypothesis that the induced velocity is uniform over the rotor:

$$
\begin{aligned}
C_{R m j} & =\sigma a\left(\frac{\mu_{j}}{8}\left(\lambda_{j}-\frac{4}{3} \theta_{0}+\theta_{t w}\right)+\frac{b_{1}}{16}\left(1-\frac{\mu_{j}^{2}}{2}\right)\right), \\
C_{T j} & =\sigma a\left(\left(1+\frac{3}{2} \mu_{j}^{2}\right) \frac{\theta_{0}}{6}-\left(1+\mu_{j}^{2}\right) \frac{\theta_{t w}}{8}-\frac{\lambda_{j}}{4}\right), \\
\lambda_{j} & =\sigma a \frac{\left(1+\frac{3}{2} \mu_{j}^{2}\right) \frac{\theta_{0}}{6}-\left(1+\mu_{j}^{2}\right) \frac{\theta_{t w}}{8}-\frac{\lambda_{j}}{4}}{2 \sqrt{\mu_{j}^{2}+\lambda_{j}^{2}}}+\frac{w_{w}-w_{j}+q l \mathrm{c}_{j}-p l \mathrm{~s}_{j}}{R\left|\omega_{j}\right|}, \\
\mu_{j} & =\frac{1}{R\left|\omega_{j}\right|} \sqrt{\left(u_{j}-u_{w}\right)^{2}+\left(v_{j}-v_{w}\right)^{2}}, \\
C_{Q_{j}} & =C_{Q_{P j}}+C_{Q_{i j}}, \quad C_{H j}=C_{H P j}+C_{H i j}, \\
\frac{C_{Q_{P j}}}{\sigma} & =\frac{1}{8}\left(C_{D 0}+C_{D i} \theta_{0}^{2}\right)\left(1+\mu_{j}^{2}\right)-C_{D i} \theta_{0} \theta_{t w}\left(\frac{1}{5}+\frac{\mu_{j}^{2}}{6}\right)+C_{D i} \theta_{t w}^{2}\left(\frac{1}{12}+\frac{\mu_{j}^{2}}{16}\right)-C_{D i} \lambda_{j} \\
& \times\left(\frac{\theta_{0}}{3}-\frac{\theta_{t w}}{4}\right)+C_{D i}\left(\frac{\mu_{j}^{2}}{8}\left(a_{0}^{2}+\frac{a_{1}^{2}}{4}+\frac{3 b_{1}^{2}}{4}\right)+\frac{1}{16}\left(a_{1}^{2}+b_{1}^{2}\right)+\frac{\lambda_{j}^{2}}{4}+\frac{\mu_{j}}{6} a_{0} a_{1}-\frac{\lambda_{j} \mu_{j}}{4} b_{1}\right), \\
\frac{C_{Q_{i j}}}{\sigma a} & =\lambda_{j}\left(\frac{\theta_{0}}{6}-\frac{\theta_{t w}}{8}-\frac{\lambda_{j}}{4}\right)-\frac{\mu_{j}^{2}}{8}\left(a_{0}^{2}+\frac{a_{1}^{2}}{4}+\frac{3 b_{1}^{2}}{4}\right)-\frac{1}{16}\left(a_{1}^{2}+b_{1}^{2}\right)-\frac{\mu_{j}}{6} a_{0} a_{1}+\frac{\lambda_{j} \mu_{j}}{4} b_{1}, \\
\frac{C_{H P j}}{\sigma} & =\frac{\mu_{j}}{4}\left(C_{D 0}+C_{D i} \theta_{0}^{2}\right)+C_{D i}\left(\frac{\mu_{j}}{24}\left(3 \theta_{t w}^{2}-8 \theta_{0} \theta_{t w}\right)+\frac{\theta_{0}}{24}\left(3 \mu_{j}^{2} b_{1}-12 \lambda_{j} \mu_{j}-4 b_{1}\right)\right. \\
& \left.-\frac{\theta_{t w}}{16}\left(\mu_{j}^{2} b_{1}-4 \lambda_{j} \mu_{j}-2 b_{1}\right)+\frac{\mu_{j}^{2}}{8} a_{0} a_{1}+\frac{\mu_{j}}{16}\left(a_{1}^{2}-b_{1}^{2}\right)+\frac{1}{4} \lambda_{j} b_{1}\right), \\
\frac{C_{H i j}}{\sigma a} & =\frac{\theta_{0}}{4}\left(\lambda_{j} \mu_{j}+\frac{2}{3} b_{1}\right)-\frac{\theta_{t w}}{8}\left(\lambda_{j} \mu_{j}+b_{1}\right)+\frac{\mu_{j}}{8}\left(a_{0}^{2}+b_{1}^{2}\right)-\frac{3}{8} \lambda_{j} b_{1}+\frac{1}{12} a_{0} a_{1},
\end{aligned}
$$

where $\left(u_{w}, v_{w}, w_{w}\right)$ are the wind velocity components in body frame in $(x, y, z)$ directions, $\sigma$ is the rotor solidity ratio, $a$ is the lift curve slope of the blade section, $C_{D 0}$ is the drag coefficient of the blade section, $C_{D i}$ is the induced drag coefficient of the blade section, $\theta_{0}$ is the angle of attack of the root profile, $\theta_{t w}$ is the blades' twist angle, $a_{0}, a_{1}, b_{1}$ are the coefficients of the blade flapping equation. The structure of the above aerodynamic coefficients can be explained by recalling aerodynamics: the thrust is the resultant of the vertical forces acting on all the blade elements. The variables $\lambda_{j}$ are the ratio between the component of vehicle velocity perpendicular to the rotor disk with respect to the blade tip speed. The variables $\mu_{j}$ indicate the component of the vehicle velocity parallel to the rotor disk with respect to the blade tip speed. The rolling moment of a propeller exists in forward flight when the advancing blade is producing more lift than the retreating one and it is the integration over the entire rotor of the lift of each section acting at a given radius. The hub force is the resultant of the horizontal forces acting on all the blade elements. The drag moment is caused by the aerodynamic forces acting on the blade elements. It is computed by multiplying these horizontal forces with the moment arm and then integrated over the rotor. As noticed, the full quadrotor model is too complex to be studied effectively in estimation theory. This complexity comes from the relation between the inflow ratio $\lambda_{j}$ and the advance ratio $\mu_{j}$. For this reason, some simplifications must be applied. Identification results at low/moderate velocity, partially illustrated by Planckaert \& Coton (2015), validate the previous vehicle model in eq. (6) and allow more simplifications to be accepted:

1) $a_{0}, a_{1}, b_{1}=0$, considering that in our case the blade flapping dynamics is characterized 
by a quick response and that the rotor's blades are stiff enough, the flapping effect is neglected.

2) $\theta_{t w}=0$ can be accepted as simplification by Planckaert $\&$ Coton (2015).

3) $C_{D i}=0$.

4) $\lambda_{j}=\lambda_{\text {stat }}-\frac{4}{\sigma a} K_{z} \frac{w_{j}-w_{w}}{R\left|\omega_{j}\right|}, K_{z}, \lambda_{\text {stat }} \geq 0$, where $K_{z}$ (as by Planckaert \& Coton (2015)) comes from the approximation of the $\lambda$ equation in vertical ascending flight, subscript stat indicates the value in hover condition.

5) $C_{T j}=C_{T_{s t a t}}+K_{z} \frac{w_{j}-w_{w}}{R\left|\omega_{j}\right|}, C_{T s t a t} \geq 0$, such models of $\lambda_{j}$ and $C_{T j}$ are rather precise in the climbing phase, but less accurate in descent phase, since the model tends to slightly overestimate the propulsion in the descent phase.

6) $C_{H j}=K_{D} \mu_{j}, K_{D} \geq 0$, the vehicle drag is modeled as $\rho A R^{2} \sum C_{H j} \omega_{j}^{2}$, corresponding to the rotors at low vehicle speed, otherwise at higher airspeed we need to add the body drag effect since it depends on square of velocity; however the constant $K_{D}$, (as by Planckaert \& Coton (2015)) has been identified for the forward velocity less than $5 \mathrm{~m} / \mathrm{s}$ taking into account the interactions between the rear and the front rotors, and considering the whole vehicle body and rotors; this term captures effects that are not easily modeled (blade flapping, interaction of rotor wakes).

Substituting the Eqs. (3), (4), (5) in the Eqs. (1), (2), and adopting the proposed identification results, the vehicle dynamics can be rewritten in state-space form

$$
\dot{\mathbf{X}}=f\left(\mathbf{X}, \mathbf{U}, \omega, \mathbf{d}_{\mathbf{w}}\right)
$$

where $f$ is expressed in the Eqs. (1), (2); $\mathbf{d}_{\mathbf{w}}=\left[u_{w} v_{w} w_{w}\right]^{T}$ is the vector of time-varying wind velocities to be estimated; $\omega$ is the vector of rotor angular velocities directly measured by the sensors; the control input $\mathbf{U}=\left[\begin{array}{llll}U_{w} & U_{p} & U_{q} & U_{r}\end{array}\right]^{T}$ contains the terms proportional to the squares of rotor velocities $\omega_{j}^{2}$ and is defined as

$$
\mathbf{U}=\left[\begin{array}{c}
U_{z} \\
U_{p} \\
U_{q} \\
U_{r}
\end{array}\right] \approx\left[\begin{array}{cccc}
K_{f} & K_{f} & K_{f} & K_{f} \\
K_{f} l \mathrm{c}_{j} & K_{f} l \mathrm{c}_{j} & K_{f} l \mathrm{c}_{j} & K_{f} l \mathrm{c}_{j} \\
-K_{f} l \mathrm{~s}_{j} & -K_{f} l \mathrm{~s}_{j} & -K_{f} l \mathrm{~s}_{j} & -K_{f} l \mathrm{~s}_{j} \\
K_{m} & -K_{m} & K_{m} & -K_{m}
\end{array}\right]\left[\begin{array}{c}
\omega_{1}^{2} \\
\omega_{2}^{2} \\
\omega_{3}^{2} \\
\omega_{4}^{2}
\end{array}\right]
$$

with $\omega_{j_{\min }} \leq \omega_{j} \leq \omega_{j_{\max }}$, where $\omega_{j}$ are the rotors' angular velocity (if $\omega_{j}<\omega_{\min }$ the rotor will stall, and $\omega_{\max }$ is given by the limitation of the rotors power), and where $K_{f}=\rho A R^{2} C_{T \text { stat }}$ and $K_{m}=\rho A R^{3}\left(\frac{\sigma C_{D 0}}{8}+\lambda_{\text {stat }} \sigma a\left(\frac{\theta_{0}}{6}-\frac{\lambda_{\text {stat }}}{4}\right)\right)$; and the state $\mathbf{X}=[u v w p q r \phi \theta \psi]^{T}$ represents the vehicle's linear and angular velocities.

\section{Decomposition in known and unknown terms}

Accelerometers measure the external forces. So, quadrotor linear body velocities $(u, v, w)$ together with their derivatives (accelerations) are provided by on-board accelerometers, which measure directly $\tilde{\Delta}_{a}(\mathbf{X})=\Delta_{a}(\mathbf{X})+\varepsilon_{\mathbf{a}}$, where $\varepsilon_{\mathbf{a}}$ is a bounded measurement noise of the accelerometer, and

$$
\Delta_{a}(\mathbf{X})=\left[\begin{array}{c}
\Delta_{a u}(\mathbf{X}) \\
\Delta_{a v}(\mathbf{X}) \\
\Delta_{a w}(\mathbf{X})
\end{array}\right]=\left[\begin{array}{c}
\dot{u} \\
\dot{v} \\
\dot{w}
\end{array}\right]+\left[\begin{array}{c}
p \\
q \\
r
\end{array}\right] \times\left[\begin{array}{c}
u \\
v \\
w
\end{array}\right]-\left[\begin{array}{c}
-g \sin \theta \\
g \cos \theta \sin \phi \\
g \cos \theta \cos \phi
\end{array}\right],
$$


where $g$ is the gravity acceleration. The remaining states are measured with the gyroscopes, which measure the rotational velocity in the body frame with respect to the Earth, as

$$
\begin{aligned}
\tilde{\Delta}_{g}(\mathbf{X}) & =\Delta_{g}(\mathbf{X})+\boldsymbol{\varepsilon}_{\mathbf{g}}, \\
\Delta_{g}(\mathbf{X}) & =[p q r]^{T},
\end{aligned}
$$

where $\varepsilon_{\mathrm{g}}$ is the measurement noise generated by gyroscopes. Accelerometers and gyroscopes augmented with an earth reference tracking system can be used to estimate $(u, v, w, \phi, \theta, \psi)$ applying a Kalman Filter, for example. The effect of the wind on the most sensors on the board of the drone (accelerometers, gyroscopes) is seen through aerodynamic forces and moments. Aerodynamic forces depend on the speed of the drone relative to the wind. It is therefore necessary to measure the speed of the drone relative to the ground (optical tracking, GPS, optical flow measurement). The use of the earth reference tracking system is twofold. First, the improvement of the estimation accuracy of $(u, v, w)$. Second, the estimation of $(\phi, \theta, \psi)$ making the drone observable with respect to the Earth frame in order to transpose the wind speeds, estimated in body frame in this paper, to the Earth frame using the rotation matrix. Following the structure used to represent the measured information, another decomposition can be performed by splitting the dynamic equations in two parts

$$
\left[\begin{array}{c}
\Delta_{a}(\mathbf{X}) \\
\dot{\Delta}_{g}(\mathbf{X})
\end{array}\right]=\mathbf{f}_{\mathbf{0}}(\mathbf{X}, \mathbf{U}, \omega)+\Omega(\omega) \mathbf{d}_{\mathbf{w}}
$$

where $\mathbf{f}_{\mathbf{0}}$ is assumed to be known (its expression is detailed below), and $\Omega$ is a time-varying regressor matrix related to the wind speed $\mathbf{d}_{\mathbf{w}}$, which has to be estimated.

Remark 1. Nonlinear terms, which represent a small part of rotor rolling torque $R_{m}$ for roll

$$
+\operatorname{sign}\left(\omega_{j}\right) \frac{1}{2} \rho A R K_{z}\left(u_{j}-u_{w}\right)\left(w_{j}-w_{w}\right),
$$

and for pitch

$$
+\operatorname{sign}\left(\omega_{j}\right) \frac{1}{2} \rho A R K_{z}\left(v_{j}-v_{w}\right)\left(w_{j}-w_{w}\right),
$$

and a small part of rotor torque $Q$ for yaw dynamics

$$
-\operatorname{sign} \omega_{j} \rho A R\left(\frac{1}{8} \sigma C_{D 0}\left(\left(u_{j}-u_{w}\right)^{2}+\left(v_{j}-v_{w}\right)^{2}\right)-\frac{1}{\sigma a} 4 K_{z}^{2}\left(w_{j}-w_{w}\right)^{2}\right)
$$

are neglected. These restrictions can be accepted for low vehicle velocity and low wind velocity, by Planckaert \& Coton (2015).

In the following, the argument dependence of the functions $\mathbf{f}_{\mathbf{0}}$ and $\Omega$ is avoided to make the 
presentation more compact:

$$
\begin{aligned}
\Delta_{a u} & =f_{0 u}+\Omega_{u} \mathbf{d}_{\mathbf{w}}, \quad f_{0 u}=-\frac{1}{m} \rho A R K_{D} \sum u_{j}\left|\omega_{j}\right|, \quad \Omega_{u} \mathbf{d}_{\mathbf{w}}=\frac{1}{m} \rho A R K_{D} u_{w} \sum\left|\omega_{j}\right| \\
\Delta_{a v} & =f_{0 v}+\Omega_{v} \mathbf{d}_{\mathbf{w}}, \quad f_{0 v}=-\frac{1}{m} \rho A R K_{D} \sum v_{j}\left|\omega_{j}\right|, \quad \Omega_{v} \mathbf{d}_{\mathbf{w}}=\frac{1}{m} \rho A R K_{D} v_{w} \sum\left|\omega_{j}\right| ; \\
\Delta_{a w} & =f_{0 w}+\Omega_{w} \mathbf{d}_{\mathbf{w}}, \quad f_{0 w}=-\frac{U_{w}}{m}-\frac{1}{m} \rho A R K_{z} \sum w_{j}\left|\omega_{j}\right|, \quad \Omega_{w} \mathbf{d}_{\mathbf{w}}=\frac{1}{m} \rho A R K_{z} w_{w} \sum\left|\omega_{j}\right| ; \\
\dot{p} & =f_{0 p}+\Omega_{p} \mathbf{d}_{\mathbf{w}}, \\
f_{0 p} & =\frac{I_{y y}-I_{z z}}{I_{x x}} q r+\frac{U_{p}}{I_{x x}}+\frac{1}{I_{x x}} \sum_{j=1}^{4}\left(\omega_{j} \rho A R^{2} u_{j} \frac{\sigma a}{2}\left(\frac{\theta_{0}}{3}-\frac{\lambda_{s t a t}}{4}\right)+\left|\omega_{j}\right| \rho A R\left(h K_{D} v_{j}-l K_{z} w_{j} \mathrm{~s}_{j}\right)\right), \\
\Omega_{p} \mathbf{d}_{\mathbf{w}} & =\frac{1}{I_{x x}} \sum_{j=1}^{4}\left(-\omega_{j} \rho A R^{2} u_{w} \frac{\sigma a}{2}\left(\frac{\theta_{0}}{3}-\frac{\lambda_{s t a t}}{4}\right)+\left|\omega_{j}\right| \rho A R\left(-h K_{D} v_{w}+l K_{z} w_{w} \mathrm{~s}_{j}\right)\right) \\
\dot{q} & =f_{0 q}+\Omega_{q} \mathbf{d}_{\mathbf{w}}, \\
f_{0 q} & =\frac{I_{z z}-I_{x x}}{I_{y y}} p r+\frac{U_{q}}{I_{y y}}+\frac{1}{I_{y y}} \sum_{j=1}^{4}\left(\omega_{j} \rho A R^{2} v_{j} \frac{\sigma a}{2}\left(\frac{\theta_{0}}{3}-\frac{\lambda_{s t a t}}{4}\right)+\left|\omega_{j}\right| \rho A R\left(l K_{z} w_{j} \mathrm{c}_{j}-h K_{D} u_{j}\right)\right), \\
\Omega_{q} \mathbf{d}_{\mathbf{w}} & =\frac{1}{I_{y y}} \sum_{j=1}^{4}\left(-\omega_{j} \rho A R^{2} v_{w} \frac{\sigma a}{2}\left(\frac{\theta_{0}}{3}-\frac{\lambda_{s t a t}}{4}\right)+\left|\omega_{j}\right| \rho A R\left(h K_{D} u_{w}-l K_{z} w_{w} \mathrm{c}_{j}\right)\right) \\
\Omega_{r} \mathbf{d}_{\mathbf{w}} & =\frac{1}{I_{z z}} \sum_{j=1}^{4}\left(-\omega_{j} \rho A R^{2} K_{z} w_{w}\left(\frac{2 \theta_{0}}{3}-2 \lambda_{s t a t}\right)-\left|\omega_{j}\right| \rho l A R K_{D}\left(-v_{w} \mathrm{c}_{j}+u_{w} \mathrm{~s}_{j}\right)\right) . \\
\dot{r} & =f_{0 r}+\Omega_{r} \mathbf{d}_{\mathbf{w}}, \\
f_{0 r} & =\frac{I_{x x}-I_{y y}}{I_{z z}} p q+\frac{U_{r}}{I_{z z}}+\frac{1}{I_{z z}} \sum_{j=1}^{4}\left(\omega_{j} \rho A R^{2} K_{z} w_{j}\left(\frac{2 \theta_{0}}{3}-2 \lambda_{s t a t}\right)-\left|\omega_{j}\right| \rho l A R K_{D}\left(v_{j} \mathrm{c}_{j}-u_{j} \mathrm{~s}_{j}\right)\right), \\
&
\end{aligned}
$$

To design the wind estimation algorithm let us consider the following system

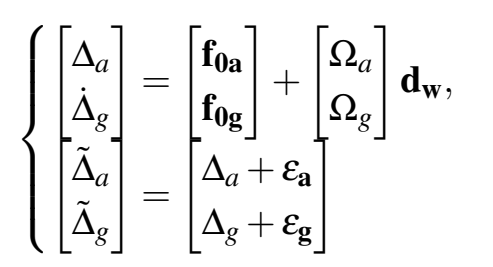

where the variables $\tilde{\Delta}_{a}$ and $\tilde{\Delta}_{g}$ are the measurements, $\mathbf{f}_{0 \mathbf{a}}=\left[f_{0 u} f_{0 v} f_{0 w}\right]^{T}, \mathbf{f}_{0 \mathbf{g}}=\left[f_{0 p} f_{0 q} f_{0 r}\right]^{T}$, $\Omega_{a}=\left[\Omega_{u} \Omega_{v} \Omega_{w}\right]^{T}$ and $\Omega_{g}=\left[\Omega_{p} \Omega_{q} \Omega_{r}\right]^{T}$ are the vector and matrix variables, whose values are functions of measured variables $\mathbf{X}, \mathbf{U}$ and $\omega$. The simplest estimation problem is to find an unknown input from the state measurements, where the input and the state are related by a first order differential equation using various differentiation schemes as in Stotsky \& Kolmanovsky (2001). However, to avoid unnecessary state differentiation, three sub-algorithms can be considered: one suitable for linear dynamics given by the accelerometers, the others for 
rotational dynamics given by the gyroscopes. The following algorithms are designed assuming the availability of the estimation of $(u, v, w)$ from the accelerometer equation, and $(\phi, \theta)$ with their derivatives. For simplicity, in theoretical analysis we assume that these values are reconstructed exactly, but for simulation a state measurement noise will be added modeling this effect. The algorithms are based on the following assumptions (we will switch between them depending on the applied approach):

Assumption 1. The measurement noises are absent $\left(\varepsilon_{\mathbf{a}}=0, \varepsilon_{\mathbf{g}}=0\right)$ and the wind velocity is constant $\left(\dot{\mathbf{d}}_{\mathbf{w}}=0\right)$.

Assumption 2. The measurement noises $\varepsilon_{\mathbf{a}}, \varepsilon_{\mathrm{g}}$ and the wind first derivative $\dot{\mathbf{d}}_{\mathbf{w}}$ are bounded signals: $\sup _{t \geq 0} \max \left\{\left|\varepsilon_{\mathbf{a}}(t)\right|,\left|\varepsilon_{\mathbf{g}}(t)\right|\right\} \leq \bar{\varepsilon}, \quad \sup _{t \geq 0}\left|\dot{\mathbf{d}}_{\mathbf{w}}(t)\right| \leq \overline{\mathbf{d}}_{\mathbf{w}}$, for some $\bar{\varepsilon}>0$ and $\overline{\mathbf{d}}_{\mathbf{w}}>0$.

Assumption 3. The matrix $\Omega_{g}$ is bounded and persistently excited (PE) for all $t \geq 0$ (see Narendra \& Annaswamy (1989)) for a definition of this property: a Lebesgue measurable and square integrable matrix function $\mathbf{R}: \mathbb{R} \rightarrow \mathbb{R}^{m \times n}$ is PE if there are $\ell>0, \xi>0$ such that $\int_{t}^{t+\ell} \mathbf{R}(\mathbf{s}) \mathbf{R}(\mathbf{s})^{T} d s \geq \xi \mathbf{I}_{\mathbf{m}}$ for all $t \geq 0$, where $\mathbf{I}_{\mathbf{m}}$ is an identity square matrix of dimension $\left.m\right)$.

\section{Estimation algorithms}

For the analysis of the time-varying system the time dependence, which was omitted before, the time dependence is made explicit. Define the estimated acceleration $\hat{\Delta}_{a}(t)=\mathbf{f}_{0 \mathbf{a}}(t)+$ $\Omega_{a}(t) \hat{\mathbf{d}}_{\mathbf{w}}(t)$, which is based on the estimate $\hat{\mathbf{d}}_{\mathbf{w}}(t)$ of the wind velocity derived below; and introduce the error $\mathbf{e}_{\mathbf{a}}(t)=\tilde{\Delta}_{a}(t)-\hat{\Delta}_{a}(t)=\Omega_{a}(t)\left(\mathbf{d}_{\mathbf{w}}(t)-\hat{\mathbf{d}}_{w}(t)\right)+\varepsilon_{\mathbf{a}}(t)$. According to Rios, Efimov, Moreno, Perruquetti, \& Rueda-Escobedo (2017) (see the equation (4) there), the following finite-time estimation algorithm can be introduced

$$
\dot{\hat{\mathbf{d}}}_{w}(t)=\gamma_{a} \Omega_{a}(t)^{T}\left\lceil\mathbf{e}_{\mathbf{a}}(t)\right\rfloor^{\alpha_{a}}, \quad 0<\alpha_{a}<1, \gamma_{a} \gg 0,
$$

where $\lceil\cdot\rfloor^{\alpha_{a}}=|\cdot|^{\alpha_{a}} \operatorname{sign}(\cdot)$ is understood elementwise. The Lyapunov function for this estimation algorithm can be selected as

$$
V(t)=\frac{1}{2 \gamma_{a}}\left|\mathbf{d}_{\mathbf{w}}(t)-\hat{\mathbf{d}}_{\mathbf{w}}(t)\right|^{2},
$$

and, with Eq. (7), its first derivative is

$$
\begin{aligned}
\dot{V}(t) & =\frac{1}{\gamma_{a}}\left(\mathbf{d}_{\mathbf{w}}(t)-\hat{\mathbf{d}}_{\mathbf{w}}(t)\right)^{T}\left(\mathbf{d}_{\mathbf{w}}(t)-\hat{\mathbf{d}}_{\mathbf{w}}(t)\right) \\
& =-\left(\mathbf{d}_{\mathbf{w}}(t)-\hat{\mathbf{d}}_{\mathbf{w}}(t)\right)^{T} \Omega_{a}(t)^{T}\left\lceil\mathbf{e}_{\mathbf{a}}(t)\right\rfloor{ }^{\alpha_{a}}+\frac{1}{\gamma_{a}}\left(\mathbf{d}_{\mathbf{w}}(t)-\hat{\mathbf{d}}_{\mathbf{w}}(t)\right)^{T} \dot{\mathbf{d}}_{\mathbf{w}}(t) \\
& =-\left(\mathbf{d}_{\mathbf{w}}(t)-\hat{\mathbf{d}}_{\mathbf{w}}(t)\right)^{T} \Omega_{a}(t)^{T}\left\lceil\Omega_{a}(t)\left(\mathbf{d}_{\mathbf{w}}(t)-\hat{\mathbf{d}}_{\mathbf{w}}(t)\right)+\varepsilon_{\mathbf{a}}(t)\right\rfloor^{\alpha_{a}}+\frac{1}{\gamma_{a}}\left(\mathbf{d}_{\mathbf{w}}(t)-\hat{\mathbf{d}}_{\mathbf{w}}(t)\right)^{T} \dot{\mathbf{d}}_{\mathbf{w}}(t) .
\end{aligned}
$$

From the identified quadrotor dynamics $\Omega_{a}(t)=\frac{1}{m} \rho A R \sum\left|\omega_{j}(t)\right| \operatorname{diag}\left\{K_{D}, K_{D}, K_{z}\right\}$, thus, since for a flying drone $\sum\left|\omega_{j}(t)\right|>0$, this is an invertible matrix, and according to Rios et al. (2017) (see the proof of Theorem 2 there) there exist two constants $v_{a 1}>0, v_{a 2}>0$ such that

$$
\dot{V}(t) \leq-v_{a 1} V(t)^{\frac{\alpha_{a}+1}{2}}, \quad \forall\left|\mathbf{d}_{\mathbf{w}}(t)-\hat{\mathbf{d}}_{\mathbf{w}}(t)\right|>v_{a 2} \max \left\{\overline{\mathbf{d}}_{\mathbf{w}}, \bar{\varepsilon}\right\}
$$


Applying Theorem 1 from Hong, Jiang, \& Feng (2010) or Corollary 2 from Rios et al. (2017) the following results have been proven:

Theorem 1. Let Assumption 1 be satisfied, then the value $\mathbf{d}_{\mathbf{w}}(t)$ can be estimated in a finite time by Eq. (7), and the estimation error $\mathbf{d}_{\mathbf{w}}(t)-\hat{\mathbf{d}}_{\mathbf{w}}(t)$ dynamics is Globally Finite-time Stable.

Theorem 2. Let Assumption 2 be satisfied, then for (7) there exist $T\left(\overline{\mathbf{d}}_{\mathbf{w}}, \bar{\varepsilon}\right)>0$ and $v_{a 2}>0$ such that

$$
\left|\mathbf{d}_{\mathbf{w}}(t)-\hat{\mathbf{d}}_{\mathbf{w}}(t)\right| \leq v_{a 2} \max \left\{\overline{\mathbf{d}}_{\mathbf{w}}, \bar{\varepsilon}\right\} \quad \forall t \geq T\left(\overline{\mathbf{d}}_{\mathbf{w}}, \bar{\varepsilon}\right),
$$

and the estimation error $\mathbf{d}_{\mathbf{w}}(t)-\hat{\mathbf{d}}_{\mathbf{w}}(t)$ dynamics is Globally Ultimate Bounded.

Therefore, the system in Eqs. (7) is globally finite-time stable, and the parameter identification error converges to a neighborhood of the origin that depends on the upper bound of the noise $\bar{\varepsilon}$, maximal amplitude of acceleration of the wind $\overline{\mathbf{d}}_{\mathbf{w}}$, the choice of the gain $\gamma_{a}$ and the parameter $\alpha_{a}$.

Wind estimation using rotational dynamics is slightly different from the linear one because only the state vector is measured but not its derivative, hence an adaptive observer, which estimates the state and the wind simultaneously, has to be also designed. To this end, first, the adaptive observer equations can be written as follows

$$
\begin{aligned}
\dot{\hat{\Delta}}_{g}(t) & =\mathbf{f}_{\mathbf{0}}(t)+\Omega_{g}(t) \hat{\mathbf{d}}_{\mathbf{w}}(t)+\ell_{g} \operatorname{sign}\left(\tilde{\Delta}_{g}(t)-\hat{\Delta}_{g}(t)\right), \\
\dot{\mathbf{d}}_{\mathbf{w}}(t) & =\gamma_{g} \Omega_{g}^{T}(t)\left(\tilde{\Delta}_{g}(t)-\hat{\Delta}_{g}(t)\right),
\end{aligned}
$$

where $\hat{\Delta}_{g}(t)$ is an estimate of the state vector $\Delta_{g}(t), \hat{\mathbf{d}}_{\mathbf{w}}(t)$ is again an estimate of the vector of wind velocities $\mathbf{d}_{\mathbf{w}}(t) ; \ell_{g}>0$ and $\gamma_{g} \gg 0$ are tuning parameters. For this observer let us consider the Lyapunov function as

$$
V(t)=\frac{1}{2}\left(\left|\Delta_{g}(t)-\hat{\Delta}_{g}(t)\right|^{2}+\frac{1}{\gamma_{g}}\left|\mathbf{d}_{\mathbf{w}}(t)-\hat{\mathbf{d}}_{\mathbf{w}}(t)\right|^{2}\right),
$$

which for (8), and under Assumption 1, allows the following derivative in time

$$
\dot{V}(t)=-\ell_{g}\left|\Delta_{g}(t)-\hat{\Delta}_{g}(t)\right| .
$$

Since $\dot{V}(t) \leq 0$ for all $t \geq 0$, the function $V(t)$ is bounded for all $t \geq 0$ and the state estimation error $\Delta_{g}(t)-\hat{\Delta}_{g}(t)$ converges asymptotically to the origin applying LaSalle invariance principle (see Theorem 14 from Desch, Logemann, Ryan, \& Sontag (2001)). The wind estimation error $\mathbf{d}_{\mathbf{w}}(t)-\hat{\mathbf{d}}_{\mathbf{w}}(t)$ converges to the origin due to the persistence of excitation in $\Omega_{g}(t)$ (see Assumption 3). Thus, let us consider an auxiliary Lyapunov function

$$
W(t)=\frac{1}{2}\left|\Delta_{g}(t)-\hat{\Delta}_{g}(t)\right|^{2},
$$

whose derivative allows an upper bound

$$
\dot{W}(t) \leq\left|\Delta_{g}(t)-\hat{\Delta}_{g}(t)\right|\left(\left|\Omega_{g}(t)\left(\mathbf{d}_{\mathbf{w}}(t)-\hat{\mathbf{d}}_{\mathbf{w}}(t)\right)\right|-\ell_{g}\right),
$$


thus, if $\ell_{g}$ is selected accordingly and $\left|\Omega_{g}(t)\left(\mathbf{d}_{\mathbf{w}}(t)-\hat{\mathbf{d}}_{w}(t)\right)\right|-\ell_{g}<-v_{g}$ for some $v_{g}>0$ (as it was shown above the signal $\Omega_{g}(t)\left(\mathbf{d}_{\mathbf{w}}(t)-\hat{\mathbf{d}}_{\mathbf{w}}(t)\right)$ is bounded), then

$$
\dot{W}(t) \leq-v_{g} \sqrt{2 W(t)}
$$

and the state estimation error $\Delta_{g}(t)-\hat{\Delta}_{g}(t)$ has a finite-time rate of convergence. The following result has been obtained (the induced norm of a matrix $\Omega_{g}(t)$ is denoted by $\left\|\Omega_{g}(t)\right\|_{2}$ ):

Theorem 3. Let Assumptions 1 and 3 be satisfied, and there is a known bound $\overline{\mathbf{d}}_{\mathbf{w}}>0$ such that $\left|\mathbf{d}_{\mathbf{w}}(t)\right| \leq \overline{\mathbf{d}}_{\mathbf{w}}$, then for $\ell_{g}>2\left\|\Omega_{g}(t)\right\|_{2} \overline{\mathbf{d}}_{\mathbf{w}}$ the estimation error $\Delta(t)-\hat{\Delta}(t)$ converges to the origin in a finite time.

The main issue with the algorithm in Eqs. (8) is hidden in rather strong restrictions imposed in Assumption 1, which we need to substantiate the convergence. Another algorithm based on less restrictive hypothesis but being more complex computationally:

$$
\begin{aligned}
\dot{\hat{\Delta}}_{g}(t) & =\mathbf{f}_{\mathbf{0 g}}(t)+\Omega_{g}(t) \hat{\mathbf{d}}_{\mathbf{w}}(t)+\ell_{g}^{\prime}\left(\tilde{\Delta}_{g}(t)-\hat{\Delta}_{g}(t)\right)+\Xi(t) \dot{\hat{\mathbf{d}}}_{\mathbf{w}}(t), \\
\dot{\Xi}(t) & =-\ell_{g}^{\prime} \Xi(t)+\Omega_{g}(t), \\
\dot{\hat{\mathbf{d}}}_{\mathbf{w}}(t) & =\gamma_{g}^{\prime} \Xi(t)^{T}\left\lceil\tilde{\Delta}_{g}(t)-\hat{\Delta}_{g}(t)\right\rfloor^{\alpha_{g}^{\prime}},
\end{aligned}
$$

where $\hat{\Delta}_{g}(t)$ and $\hat{\mathbf{d}}_{\mathbf{w}}(t)$ as before are the estimates of $\Delta_{g}(t)$ and $\mathbf{d}_{\mathbf{w}}(t)$, respectively; $\ell_{g}^{\prime}>0$, $\alpha_{g}^{\prime} \in(0,1)$ and $\gamma_{g}^{\prime} \gg 0$ are design parameters; $\Xi(t)$ is an auxiliary matrix variable having the dimension of $\Omega_{g}(t)$ (obviously it is always bounded for bounded $\Omega_{g}(t)$ and $\ell_{g}^{\prime}>0$ ). In order to clarify the stability and robustness properties of this estimation scheme let us introduce three estimation errors

$$
\mathbf{e}_{\Delta}(t)=\Delta_{g}(t)-\hat{\Delta}_{g}(t), \quad \mathbf{e}_{\mathbf{d}}(t)=\mathbf{d}_{\mathbf{w}}(t)-\hat{\mathbf{d}}_{\mathbf{w}}(t), \quad \delta(t)=\mathbf{e}_{\Delta}(t)-\Xi(t) \mathbf{e}_{\mathbf{d}}(t),
$$

which have the following dynamics

$$
\begin{aligned}
\dot{\mathbf{e}}_{\Delta}(t) & =-\ell_{g}^{\prime}\left(\mathbf{e}_{\Delta}(t)+\varepsilon_{g}(t)\right)+\Omega_{g}(t) \mathbf{e}_{\mathbf{d}}(t)-\gamma_{g}^{\prime} \Xi(t) \Xi(t)^{T}\left\lceil\mathbf{e}_{\Delta}(t) \varepsilon_{\mathbf{g}}(t)\right\rfloor^{\alpha_{g}^{\prime}}, \\
\dot{\delta}(t) & =-\ell_{g}^{\prime}\left(\boldsymbol{\delta}(t)+\varepsilon_{\mathbf{g}}(t)\right)-\Xi(t) \dot{\mathbf{d}}_{\mathbf{w}}(t), \\
\dot{\mathbf{e}}_{\mathbf{d}}(t) & =-\gamma_{g} \Xi(t)^{T}\left\lceil\Xi(t) \mathbf{e}_{\mathbf{d}}(t)+\delta(t)+\varepsilon_{\mathbf{g}}(t)\right\rfloor^{\alpha_{g}^{\prime}}+\dot{\mathbf{d}}_{\mathbf{w}}(t) .
\end{aligned}
$$

Introduce the following additional assumptions:

Assumption 4. The minimum singular value of the matrix variable $\Xi(t) \in \mathbb{R}^{3 \times 3}$ is larger than $\sigma_{\Xi}>0$ for all $t \geq 0$.

The last condition on $\Xi$ can be ensured by a proper initialization and the same property of $\Omega_{g}$ (Assumption 3). Under this assumption boundedness of all estimation errors can be proven by analyzing the independent Lyapunov functions $V(\boldsymbol{\delta}(t)), V\left(\mathbf{e}_{\mathbf{d}}(t)\right)$ and $V\left(\mathbf{e}_{\Delta}(t)\right)$, where

$$
V(\mathbf{x}(t))=0.5 \mathbf{x}(t)^{T} \mathbf{x}(t) .
$$

Indeed, under Assumption 2, first, let us analyze the behavior of $V(t)$ for the dynamics of $\delta(t)$, 
where all inputs $\left(\Xi(t), \dot{\mathbf{d}}_{\mathbf{w}}(t)\right.$ and $\left.\varepsilon_{\mathbf{g}}(t)\right)$ are bounded

$$
V \dot{(t)} \leq-0.5 \ell_{g}^{\prime} \delta^{T}(t) \delta(t)+\frac{1}{2 \ell_{g}^{\prime}}\left|\Xi(t) \dot{\mathbf{d}}_{\mathbf{w}}(t)+\ell_{g}^{\prime} \varepsilon_{\mathbf{g}}(t)\right|^{2},
$$

which implies boundedness of $\delta(t)$. Second, for the dynamics of $\mathbf{e}_{\mathbf{d}}(t)$ :

$$
\dot{V}(t) \leq-\gamma_{g}^{\prime} \mathbf{e}_{\mathbf{d}}(t)^{T} \Xi(t)^{T}\left\lceil\Xi(t) \mathbf{e}_{\mathbf{d}}(t)+\delta(t)+\varepsilon_{\mathbf{g}}(t)\right\rfloor^{\alpha_{g}^{\prime}}+\mathbf{e}_{\mathbf{d}}(t)^{T} \dot{\mathbf{d}}_{\mathbf{w}}(t),
$$

if we assume that $\left|\Xi(t) \mathbf{e}_{\mathbf{d}}(t)\right|>\left|\delta(t)+\varepsilon_{\mathbf{g}}(t)\right|$ (which is true if $\left.\left|\mathbf{e}_{\mathbf{d}}(t)\right|>\sigma_{\Xi}^{-1}\left|\delta(t)+\varepsilon_{\mathbf{g}}(t)\right|\right)$ then

$$
\begin{gathered}
\mathbf{e}_{\mathbf{d}}(t)^{T} \Xi(t)^{T}\left\lceil\Xi(t) \mathbf{e}_{\mathbf{d}}(t)+\delta(t)+\varepsilon_{\mathbf{g}}(t)\right\rfloor^{\alpha_{g}^{\prime}}=\sum_{i=1}^{3}\left|\left(\Xi(t) e_{d}(t)\right)_{i}\right|\left|\left(\Xi(t) e_{d}(t)\right)_{i}+\delta(t)_{i}+\varepsilon_{g}(t)_{i}\right|^{\alpha_{g}^{\prime}} \\
\geq 2^{\alpha_{g}^{\prime}-1} \sum_{i=1}^{3}\left|\left(\Xi(t) e_{d}(t)\right)_{i}\right|^{\alpha_{g}^{\prime}+1}+\left|\left(\Xi(t) e_{d}(t)\right)_{i}\right|\left|\delta(t)_{i}+\varepsilon_{g}(t)_{i}\right|^{\alpha_{g}^{\prime}} \\
\mathbf{e}_{\mathbf{d}}(t)^{T} \dot{\mathbf{d}}_{\mathbf{w}}(t) \leq \frac{1}{\alpha_{g}^{\prime}+1}\left|c \Xi(t) \mathbf{e}_{\mathbf{d}}(t)\right|^{\alpha_{g}^{\prime}+1}+\frac{\alpha_{g}^{\prime}}{\alpha_{g}^{\prime}+1}\left|c^{-1} \Xi(t)^{-1} \dot{\mathbf{d}}_{\mathbf{w}}(t)\right|^{1+\alpha_{g}^{\prime-1}}
\end{gathered}
$$

where $c=2^{\alpha_{g}^{\prime}-2} \gamma_{g}^{\prime}>0$, and Jensen's and Young's inequalities have been used. Thus

$$
\dot{V}(t) \leq-2^{\alpha_{g}^{\prime}-2} \gamma_{g}^{\prime}\left|\Xi(t) \mathbf{e}_{\mathbf{d}}(t)\right|^{\alpha_{g}^{\prime}+1}+\frac{\alpha_{g}^{\prime}}{\alpha_{g}^{\prime}+1}\left|c^{-1} \Xi(t)^{-1} \dot{\mathbf{d}}_{\mathbf{w}}(t)\right|^{1+\alpha_{g}^{\prime-1}}
$$

is satisfied for $\left|\Xi(t) \mathbf{e}_{\mathbf{d}}(t)\right|>\left|\delta(t)+\varepsilon_{\mathbf{g}}(t)\right|$, or equivalently

$$
\dot{V}(t) \leq-2^{\alpha_{g}^{\prime}-3} \gamma_{g}^{\prime}\left|\Xi(t) \mathbf{e}_{\mathbf{d}}(t)\right|^{\alpha_{g}^{\prime}+1} \leq-2^{\frac{\alpha_{g}^{\prime}-7}{2}} \gamma_{g}^{\prime} \sigma_{\Xi}^{1+\alpha_{g}^{\prime}} V(t)^{\frac{\alpha_{g}^{\prime}+1}{2}}
$$

provided that $\left|\mathbf{e}_{\mathbf{d}}(t)\right|>\sigma_{\Xi}^{-1} \max \left\{\left|\delta(t)+\varepsilon_{\mathbf{g}}(t)\right|, \sqrt[\alpha_{g}^{\prime}+1]{\frac{2 \alpha_{g}^{\prime}}{\alpha_{g}^{\prime}+1} c^{-2-\alpha_{g}^{\prime-1}}\left|\Xi(t)^{-1} \dot{\mathbf{d}}_{\mathbf{w}}(t)\right|^{1+\alpha_{g}^{\prime-1}}}\right\}$. Next, again boundedness of all inputs $\left(\delta(t), \varepsilon_{\mathbf{g}}(t)\right.$ and $\left.\dot{\mathbf{d}}_{\mathbf{w}}(t)\right)$ implies the same property for $\mathbf{e}_{\mathbf{d}}(t)$. Finally, for the dynamics of $\mathbf{e}_{\Delta}(t)$ :

$\dot{V}(t) \leq-\ell_{g}^{\prime} \mathbf{e}_{\Delta}(t)^{T} \mathbf{e}_{\Delta}(t)+\mathbf{e}_{\Delta}(t)^{T}\left(\Omega_{g}(t) \mathbf{e}_{\mathbf{d}}(t)+\ell_{g}^{\prime} \varepsilon_{\mathbf{g}}(t)\right)-\gamma_{g}^{\prime} \mathbf{e}_{\Delta}(t)^{T} \Xi(t) \Xi(t)^{T}\left\lceil\mathbf{e}_{\Delta}(t)+\varepsilon_{g}(t)\right\rfloor^{\alpha_{g}^{\prime}}$, and assuming that $\left|\mathbf{e}_{\Delta}(t)\right|>\sigma_{\Xi}^{-1}\left|\varepsilon_{\mathbf{g}}(t)\right|$ we obtain

$$
\dot{V}(t) \leq-0.5 \ell_{g}^{\prime} \mathbf{e}_{\Delta}(t)^{T} \mathbf{e}_{\Delta}(t)+\frac{1}{2 \ell_{g}^{\prime}}\left|\Omega_{g}(t) \mathbf{e}_{\mathbf{d}}(t)+\ell_{g}^{\prime} \varepsilon_{\mathbf{g}}(t)\right|^{2},
$$

or equivalently

$$
\dot{V}(t) \leq-0.25 \ell_{g}^{\prime} \mathbf{e}_{\Delta}(t)^{T} \mathbf{e}_{\Delta}(t)
$$

provided that $\left|\mathbf{e}_{\Delta}(t)\right|>\max \left\{\sigma_{\Xi}^{-1}\left|\varepsilon_{\mathbf{g}}(t)\right|, \frac{\sqrt{2}}{\ell_{g}^{\prime}}\left|\Omega_{g}(t) \mathbf{e}_{\mathbf{d}}(t)+\ell_{g}^{\prime} \varepsilon_{\mathbf{g}}(t)\right|\right\}$, therefore $\mathbf{e}_{\Delta}(t)$ is also bounded and the following result has been proven: 
Theorem 4. Let assumptions 2 and 4 be satisfied, then in Eqs. (9) there exists $T>0$ such that

$$
\left|\mathbf{e}_{\mathbf{d}}(t)\right| \leq \rho\left(\bar{\varepsilon}, \overline{\mathbf{d}}_{\mathbf{w}}\right), \quad\left|\mathbf{e}_{\Delta}(t)\right| \leq \max \left\{\sigma_{\Xi}^{-1} \bar{\varepsilon}, \frac{\sqrt{2}}{\ell_{g}^{\prime}}\left(\left\|\Omega_{g}(t)\right\|_{2} \rho\left(\bar{\varepsilon}, \overline{\mathbf{d}}_{\mathbf{w}}\right)+\ell_{g}^{\prime} \bar{\varepsilon}\right)\right\},
$$

for all $t \geq T$, where

$$
\rho\left(\bar{\varepsilon}, \overline{\mathbf{d}}_{\mathbf{w}}\right)=\sigma_{\Xi}^{-1} \max \left\{\frac{2}{\ell_{g}^{\prime}}\|\Xi(t)\|_{2} \overline{\mathbf{d}}_{\mathbf{w}}+3 \bar{\varepsilon}, \sqrt[\alpha_{g}^{\prime}+1]{c^{\prime} \overline{\mathbf{d}}_{\mathbf{w}}{ }^{1+\alpha_{g}^{\prime-1}}}\right\} ; \quad c^{\prime}=\frac{2 \alpha_{g}^{\prime}}{\alpha_{g}^{\prime}+1} c^{-2-\alpha_{g}^{\prime-1}} \sigma_{\Xi}^{-1-\alpha_{g}^{\prime-1}} .
$$

If $\delta(t)=\bar{\varepsilon}=\overline{\mathbf{d}}_{\mathbf{w}}=0$, then a finite-time convergence of $\hat{\mathbf{d}}_{\mathbf{w}}(t)$ to $\mathbf{d}_{\mathbf{w}}(t)$ is substantiated (this conclusion can be obtained applying Theorem 1 from Hong et al. (2010) and using $V\left(\mathbf{e}_{\mathbf{d}}(t)\right)$ as an input-to-state Lyapunov function).

The restrictions used for the estimation algorithms are not the same. Algorithm in Eqs. (8) is obtained in the noise-free and constant wind conditions. Despite the theoretical assumptions this algorithm also possesses some noise filtering abilities, however, since the simulations show that a good estimation for varying wind velocities with high frequencies is not achieved, we have decided to avoid it in the fusion algorithm. The estimation schemes in Eq. (7), and in Eqs. (9) assume both that the noise and wind derivative are bounded signals (note that the worst-case upper bounds on the estimation errors are also obtained for these algorithms). Hence, some fusion of these solutions is desirable. To this end, let us define the estimates of $\mathbf{d}_{\mathbf{w}}(t)$ generated by the Eq. (7), and Eqs. (9), as $\hat{\mathbf{d}}_{\mathbf{w}}(t)^{i}$ for $i=1,2$ respectively. Denote the errors as $v_{\mathbf{1}}(t)=\mathbf{e}_{\mathbf{a}}(t)$ for the algorithm in Eq. (7) and $v_{\mathbf{2}}(t)=\tilde{\Delta}_{g}(t)-\hat{\Delta}_{g}(t)$ for the algorithm in Eqs. (9). Then

$$
\hat{\mathbf{d}}_{\mathbf{w}}(t)^{\mathrm{fusion}}=\frac{\sum_{i=1}^{2} e^{-\kappa_{i} v_{i}(t)^{2}} \hat{\mathbf{d}}_{\mathbf{w}}(t)^{i}}{\sum_{i=1}^{2} e^{-\kappa_{i} v_{i}(t)^{2}}}
$$

is the united estimate of $\mathbf{d}_{\mathbf{w}}(t)$ from all the estimation algorithms, and $\kappa_{i}>0$ for $i=1,2$ are tuning parameters. Fusion algorithm has the estimation error, in the worst case, given by the maximum of the two estimation errors for the algorithms (7) and (9).

\section{Numerical validation}

The quadrotor parameters are illustrated in Table 1. The identification process, which is not the contribution of this paper, is described in Planckaert \& Coton (2015). Quadrotor models with two different levels of complexity are used. The quadrotor model based on the identification work is used to build the algorithms, and the full quadrotor model coming from the complete aerodynamic Eqs. (6) is used to test the effect of the avoided nonlinearities for the inflow ratio. Gaussian noises with $2.5 \mathrm{deg} / \mathrm{s}$, and $0.052 \mathrm{~m} / \mathrm{s}^{2}$ standard deviations for gyroscopes and accelerometers respectively, are added to simulate the augmented-IMU sensor's noise. Additional Gaussian noises of $1 \mathrm{~cm} / \mathrm{s}$ standard deviation for linear velocities, and $1 \mathrm{deg}$ standard deviation for angles are added because state measurements $(u, v, w, \phi, \theta)$ are not reconstructed exactly. These values come from the available cameras in the lab where the previous identification experiments were carried out. The estimation algorithms, presented in this work, can be also extended to a more realistic case without optical tracking system and considering GPS. The used quadrotor model was identified in case of the flight of the drone in the laboratory equipped with cameras which simplifies very much the disturbance estimation 


\begin{tabular}{|c|c|c|c|c|c|c|c|c|c|}
\hline $\begin{array}{c}\text { Param. } \\
\text { Values } \\
\text { Units }\end{array}$ & $\begin{array}{c}R \\
0.1 \\
m\end{array}$ & $\begin{array}{c}l \\
0.185 \\
m\end{array}$ & $\begin{array}{c}h \\
-0.025 \\
m\end{array}$ & $\begin{array}{c}g \\
9.81 \\
m / s^{2}\end{array}$ & $\begin{array}{c}\theta_{0} \\
23.9 \\
\text { deg }\end{array}$ & $\begin{array}{c}m \\
0.472 \\
k g\end{array}$ & $\begin{array}{c}I_{x x} \\
0.00356 \\
k g . m^{2}\end{array}$ & $\begin{array}{c}I_{y y} \\
0.00402 \\
k g . m^{2}\end{array}$ & $\begin{array}{c}I_{z z} \\
0.00712 \\
\text { kg. } \mathrm{m}^{2}\end{array}$ \\
\hline $\begin{array}{c}\text { Param. } \\
\text { Values }\end{array}$ & $\begin{array}{c}\rho \\
1.25\end{array}$ & $\begin{array}{c}\sigma \\
0.111\end{array}$ & $\begin{array}{c}a \\
4.6542\end{array}$ & $\begin{array}{l}C_{D 0} \\
2.15\end{array}$ & $\begin{array}{c}\lambda_{\text {stat }} \\
0.1056\end{array}$ & $\begin{array}{c}C_{T_{\text {stat }}} \\
0.0223\end{array}$ & $\begin{array}{c}K_{D} \\
0.06\end{array}$ & $\begin{array}{c}K_{z} \\
0.09\end{array}$ & $\begin{array}{c}b \\
0.1\end{array}$ \\
\hline Units & $\mathrm{kg} / \mathrm{m}^{3}$ & - & - & - & - & - & - & - & - \\
\hline
\end{tabular}

Table 2. Parameters for the wind estimators.

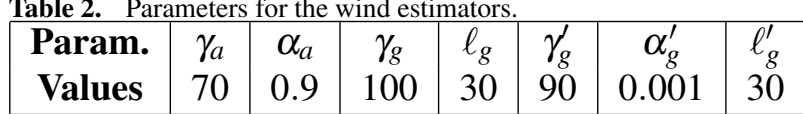

problem because it allows obtaining an immediate estimation of the speed with a high accuracy. A GPS system is less accurate and does not provide information on the attitude of the drone, and a lower precision of the location will cause some undesirable effects such as phase shift of the estimate of the state. If the estimation based on the quadrotor rotational dynamics cannot be considered, an estimation of the wind speed can be achieved with only the 3 dynamics coming from the accelerometers. Since the behavior of the vehicle is strongly dependent on the speed relative to the wind, an optical flow sensor (ground speed measurement made on board the drone) can also be used especially in case of data link breakage, but it requires to know well the attitude of the drone. The wind estimation algorithms have been designed taking into account the performance criteria which are the robustness against the sensor's noises and the finite-time estimation in short time. For simplicity of presentation, the notations algorithm-lin, algorithm-rot and algorithm-rot-2 are used respectively for algorithms in Eq. (7), in Eq. (8), and in Eq. (9).

A good algorithm must perform the estimation in a very short time to be useful for quadrotors in order to react properly to the fast quadrotor dynamics. Acceptable trade-off between accuracy and filtering are observed with the tuned parameters in Table 2, which are used for all the simulations. This trial and error tuning process is illustrated with some examples, enlightening the relation between the convergence time, the accuracy and the filtering. Fig. 1 shows a comparison for the algorithm-lin, where $\gamma_{a}=70$ is a compromise between accuracy and fast response, and a comparison for the algorithm-rot-2, which presents an undesired overshoot in the case of fast response. These results have demonstrated that more filtering ability can be obtained at the price of less accuracy in estimation and vice-versa. Based on this study, the algorithm using the linear quadrotor dynamics performs the estimation in the shortest time, while the fusion algorithm has the best filtering ability keeping an acceptable finite-time estimation, as in Fig. 2. Algorithm-rot is worse because it is slower and it is subjected to the highest uncertainty. These figures show that the estimation algorithms can achieve estimation in a shorter time in a realistic case, where the changes of the wind speeds are smoother.

For validation of the proposed algorithms, two cases are considered. The first case represents an indoor wind estimation, where the wind velocity is artificially generated by fans. The second case represents an outdoor wind estimation, where the wind profile is always unpredictable.

In the indoor case, the wind speeds may have sinusoidal dynamics with $(2 \mathrm{~m} / \mathrm{s}, 2 \mathrm{~m} / \mathrm{s}, 0.2 \mathrm{~m} / \mathrm{s})$ maximal values in $(x, y, z)$ directions and $0.3 \mathrm{rad} / \mathrm{s}$ maximal frequency, since the quadrotor identification work by Planckaert \& Coton (2015) is valid for 


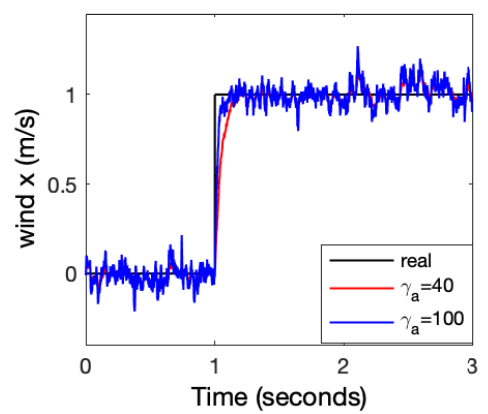

((a)) Tuning for algorithm-lin.

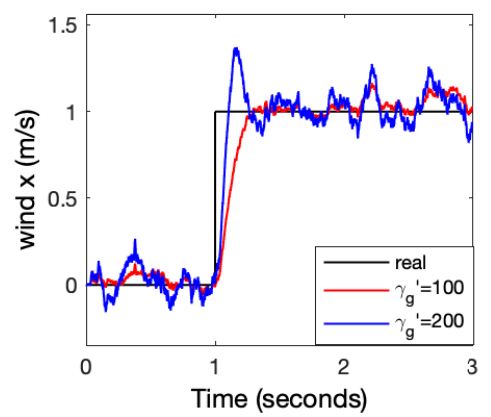

((b)) Tuning for algorithm-rot-2.

Figure 1. Tuning process comparisons for the different algorithms.

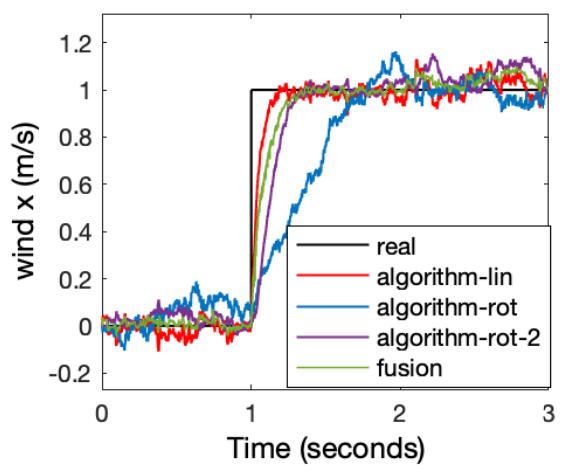

Figure 2. Comparison of the algorithms' performance for unitary step response. 
$\approx( \pm 5, \pm 5, \pm 1)$ translational velocities respectively in $(x, y, z)$ axes. For conceptual validation one simulation is performed, using the provided identified quadrotor model. Since algorithmrot and algorithm-rot-2 cannot estimate correctly the $z$ component of the wind because $w_{w}$ (wind velocity along $z$ axis) is not present in the equations of moments, then only $(x, y)$ components are considered. Fig. 3 shows: the performance of the algorithm-lin, where wind is well estimated; the algorithm-rot, where the assumed noise-free and constant wind velocity conditions influence very much the quality of the result; the algorithm-rot-2 where the problem of these restrictive hypotheses is solved; the fusion algorithm, which estimates correctly the wind velocity using a fusion of algorithm-lin and algorithm-rot-2 along the $x, y$ axes, and using only algorithm-lin for $z$ axis. It's worth to note that the estimation using algorithm fusion on $x, y$ are less noisy than the estimation using only algorithm-lin. Having performed the wind estimation for such a reference wind, it is safe to assume that the algorithms can achieve the estimation of wind having frequencies lower than the considered case and having equal maximal amplitude. Fig. 4 shows the results testing the estimation algorithm on the full nonlinear quadrotor model in the horizontal plane. Tests on the vertical directions are avoided because of the lower accuracy in the identification process due to the coefficient of thrust $C_{T}$, which is quite a challenge to precisely estimate (see Planckaert \& Coton (2015)).

In the outdoor case, the wind speeds may have $(2 \mathrm{~m} / \mathrm{s}, 2 \mathrm{~m} / \mathrm{s})$ maximal values in the horizontal plane. The wind profile is created using the Wind Field Simulation in Cheynet (2020). Fig. 5 shows the results testing the estimation algorithm on the full nonlinear quadrotor model in the horizontal plane. The presented numeric experiments show that the estimation algorithms perform well using the provided identified model for unpredictable and fast-changing wind speeds that can happens in an outdoor real scenario.

\section{Conclusion}

Considering a detailed aerodynamic model of quadrotors, three wind estimation algorithms are proposed. The analytic conditions of their applicability are established, and their robustness is analyzed. The presented numeric experiments show that the estimation algorithms perform well using the provided identified model. Experiments have also demonstrated that smaller convergence time can be obtained at the price of smaller filtering ability against noises. The algorithm based on linear quadrotor dynamics performs the estimation in the shortest time, while the fusion algorithm has the best filtering ability keeping an acceptable finite-time estimation. A final test with the full nonlinear quadrotor model shows that the airspeed estimation performs quite well in the horizontal plane.

As a direction of further development, an additional identification process on the quadrotor model can be made to improve the performance of the algorithms, avoiding the simplifications for the rotational quadrotor dynamics at low wind quadrotor speeds. The proposed estimation algorithms are designed to work on the quadrotor model with the nominal values of the identified aerodynamic coefficients. An interval estimation algorithm for the quadrotor dynamics, whose accuracy is proportional to the uncertitudes of the aerodynamic coefficients, can be used in parallel to the proposed wind estimation algorithms. 

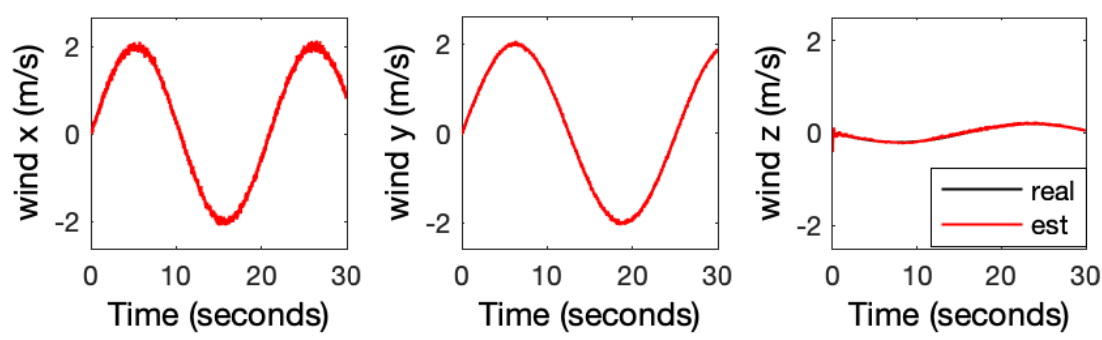

((a)) Wind estimation using algorithm-lin.
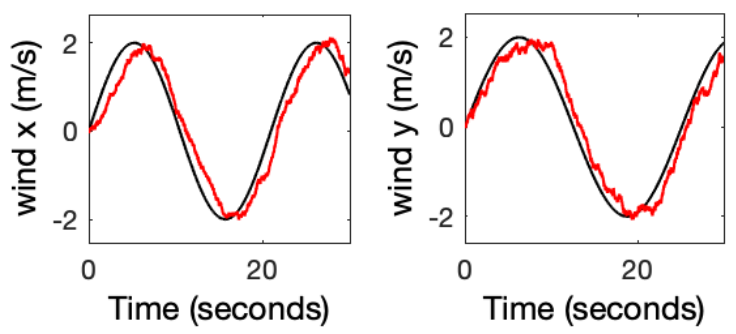

((b)) Wind estimation using using algorithm-rot.
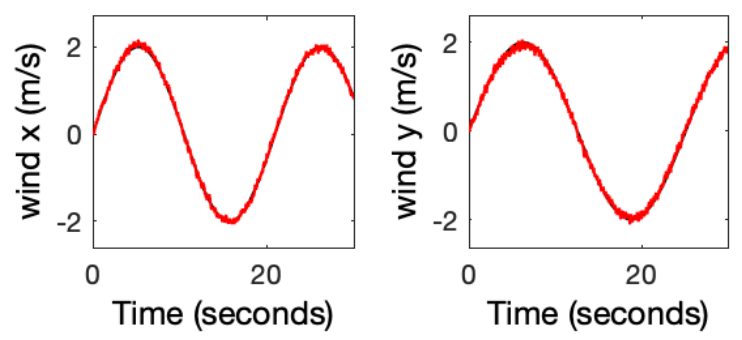

((c)) Wind estimation using algorithm-rot-2.
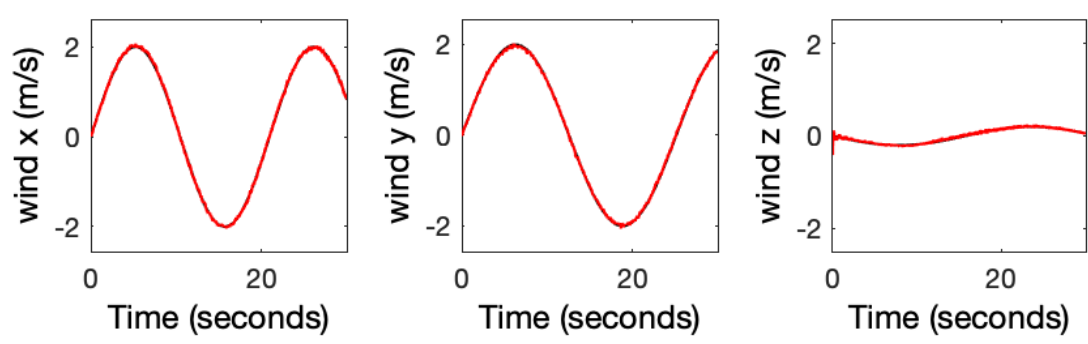

((d)) Wind estimation using algorithm-fusion.

Figure 3. Wind estimation performance using the four algorithms in indoor case.
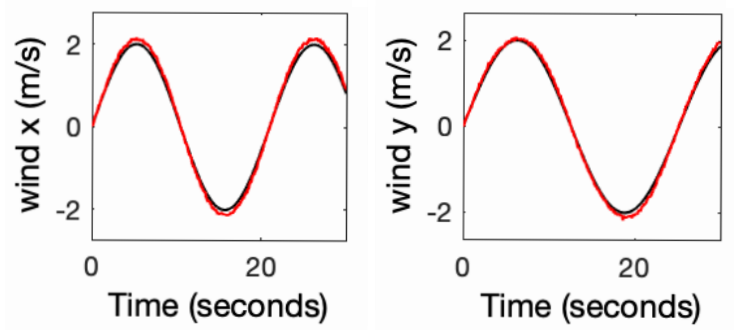

Figure 4. Wind estimation using nonlinear quadrotor model in indoor case. 

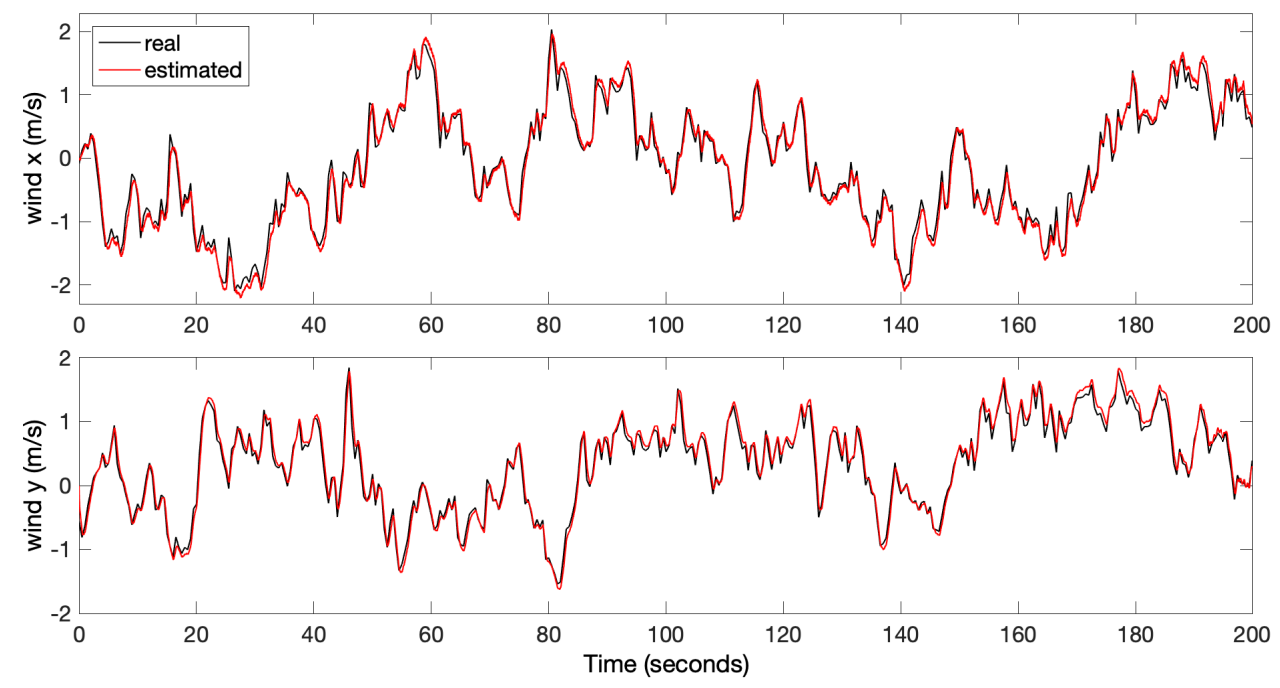

Figure 5. Wind estimation using nonlinear quadrotor model in outdoor case.

\section{Appendix}

Consider a time-dependent differential equation (see Khalil (2002)):

$$
\frac{d x(t)}{d t}=f(t, x(t)), t \geq t_{0}, t_{0} \in \mathbb{R}
$$

where $x(t) \in \mathbb{R}^{n}$ is the state vector; $f: \mathbb{R} \times \mathbb{R}^{n} \rightarrow \mathbb{R}^{n}$ is a continuous function with respect to $x \in \mathbb{R}^{n}$ and piece-wise continuous with respect to $t \in \mathbb{R}, f(0, t)=0$ for all $t \in \mathbb{R}$. The solution of the system (11) for an initial condition $x_{0} \in \mathbb{R}^{n}$ at time instant $t_{0} \in \mathbb{R}$ is denoted as $x\left(t, t_{0}, x_{0}\right)$ and defined on some finite-time (FT) interval $\left[t_{0}, t_{0}+T\right)$, we assume its existence and uniqueness locally in time for (11). Let $\Omega$ be an open neighborhood of the origin in $\mathbb{R}^{n}$, $0 \in \Omega$.

Definition (see Rios et al. (2017)): At the steady state $x=0$ the system (11) is said to be

1) Uniformly stable (US) if for any $\varepsilon>0$ there is $\delta(\varepsilon)$ such that for any $x_{0} \in \Omega$, if $\left\|x_{0}\right\| \leq \delta(\varepsilon)$ then $\left\|x\left(t, t_{0}, x_{0}\right)\right\| \leq \varepsilon$ for all $t \geq t_{0}$, for any $t_{0} \in \mathbb{R}$

2) Uniformly finite-time stable (UFTS) if it is US and FT converging from $\Omega$, i.e., for any $x_{0} \in \Omega$, there exist $0 \leq T_{x_{0}}$ such that $x\left(t, t_{0}, x_{0}\right)=0$ for all $t \geq t_{0}+T_{x_{0}}$ for any $t_{0} \in \mathbb{R}$. The function $T_{0}\left(x_{0}\right)=\inf \left\{T_{x_{0}} \geq 0: x\left(t, t_{0}, x_{0}\right)=0 \quad \forall t \geq t_{0}+T_{x_{0}}\right\}$ is called the settling-time of the system (11).

If $\Omega=\mathbb{R}^{n}$, then $x=0$ is said to be globally US/UFTS.

\section{Funding}

This work was equally funded by the region Hauts-de-France and by ONERA: The French Aerospace Lab. 


\section{References}

Ali, S. U., Shah, M. Z., Samar, R., \& Waseem, A. (2016, January). Wind estimation for lateral path following of UAVs using higher order sliding mode. In 2016 International Conference on Intelligent Systems Engineering (ICISE) (pp. 364-371).

Benallegue, A., Mokhtari, A., \& Fridman, L. (2008, March). High-order sliding-mode observer for a quadrotor UAV. International Journal of Robust and Nonlinear Control, 18(4-5), 427-440.

Boiko, I., \& Chehadeh, M. (2018, September). Sliding mode differentiator/observer for quadcopter velocity estimation through sensor fusion. International Journal of Control, 91(9), 2113-2120.

Bramwell, A. R. S., Balmford, D., \& Done, G. (2001). Bramwell's Helicopter Dynamics, second edition. Butterworth-Heinemann. (ch 2-7)

Cheynet, E. (2020, February). Wind field simulation (the user-friendly version) [https://www.mathworks.com/matlabcentral/fileexchange/50041-wind-field-simulation-theuser-friendly-version].

Demitrit, Y., Verling, S., Stastny, T., Melzer, A., \& Siegwart, R. (2017, May). Model-based wind estimation for a hovering VTOL tailsitter UAV. In 2017 IEEE International Conference on Robotics and Automation (ICRA) (pp. 3945-3952).

Desch, W., Logemann, H., Ryan, E. P., \& Sontag, E. D. (2001, June). Meagre functions and asymptotic behaviour of dynamical systems. Nonlinear Analysis: Theory, Methods \& Applications, 44(8), 1087-1109.

Gonzalez-Rocha, J., Woolsey, C. A., Sultan, C., de Wekker, S., \& Rose, N. (2017, January). Measuring Atmospheric Winds from Quadrotor Motion. In AIAA Atmospheric Flight Mechanics Conference. American Institute of Aeronautics and Astronautics.

Hong, Y., Jiang, Z.-P., \& Feng, G. (2010, January). Finite-Time Input-to-State Stability and Applications to Finite-Time Control Design. SIAM Journal on Control and Optimization, 48(7), 4395-4418.

Johnson, W. (2012). Helicopter Theory. Courier Corporation. (Chapters 2-5,9-13,15)

Karvonen, T. (2014). Stability of linear and non-linear Kalman filters (Unpublished master's thesis). University of Helsinki.

Khalil, H. K. (2002). Nonlinear Systems. NJ, USA: Prentice Hall, Englewood Cliffs.

Leishman, G. J. (2006). Principles of Helicopter Aerodynamics. Cambridge University Press. (ch 2-5)

Lie, F. A. P., \& Gebre-Egziabher, D. (2013, May). Synthetic Air Data System. Journal of Aircraft, 50(4), 1234-1249.

Martínez-Vásquez, A., Rodriguez-Mata, A., González-Hernández, I., Salazar, S., Montiel-Varela, A., \& Lozano, R. (2015, October). Linear observer for estimating wind gust in UAV's. In 2015 12th International Conference on Electrical Engineering, Computing Science and Automatic Control (CCE).

Narendra, K. S., \& Annaswamy, A. M. (1989). Stable adaptive systems. NJ, USA: Englewood Cliffs, Prentice Hall.

Neumann, P. P., \& Bartholmai, M. (2015, November). Real-time wind estimation on a micro unmanned aerial vehicle using its inertial measurement unit. Sensors and Actuators A: Physical, 235, 300-310.

Ning, C., He, Y., Wu, M., \& She, J. (2014, July). Improved Razumikhin-Type Theorem for Input-ToState Stability of Nonlinear Time-Delay Systems. IEEE Transactions on Automatic Control, 59(7), 1983-1988.

Pappu, V. S. R., Liu, Y., Horn, J. F., \& Cooper, J. (2017, January). Wind gust estimation on a small VTOL UAV. In 7th ahs technical meeting on vtol unmanned aircraft systems and autonomy.

Pendleton, D. I., \& Zhang, W. (2017, January). Development of a New Wind Measurement Tool based on a Hovering Drone. In 55th AIAA Aerospace Sciences Meeting. American Institute of Aeronautics and Astronautics.

Peng, S., \& Zhang, Y. (2010, December). Some New Criteria on \$p\$th Moment Stability of Stochastic Functional Differential Equations With Markovian Switching. IEEE Transactions on Automatic Control, 55(12), 2886-2890.

Planckaert, L., \& Coton, P. (2015, September). Quadrotor UAV aerodynamic model identification using indoor flight experiment and feasibility of UAV as wind gust sensor. In International Micro Air Vehicles Conference and Flight Competition IMAV 2015. Aachen, Germany. 
Qu, Y., Xing, Z., \& Zhang, Y. (2016, August). Wind estimation using the position information from a hovering quadrotor. In 2016 IEEE Chinese Guidance, Navigation and Control Conference (CGNCC) (pp. 1345-1350).

Qu, Y., Xing, Z., Zhang, Y., \& Yu, Z. (2017, June). Real-time wind vector estimation for a micro UAV. In 2017 International Conference on Unmanned Aircraft Systems (ICUAS) (pp. 1716-1721).

Rhudy, M. B., Fravolini, M. L., Gu, Y., Napolitano, M. R., Gururajan, S., \& Chao, H. (2015, July). Aircraft model-independent airspeed estimation without pitot tube measurements. IEEE Transactions on Aerospace and Electronic Systems, 51(3), 1980-1995.

Rios, H., Efimov, D., Moreno, J. A., Perruquetti, W., \& Rueda-Escobedo, J. G. (2017, July). TimeVarying Parameter Identification Algorithms: Finite and Fixed-Time Convergence. IEEE Transactions on Automatic Control, 62(7), 3671-3678.

Santosuosso, G. L., Benzemrane, K., \& Damm, G. (2011, November). Nonlinear speed estimation of a GPS-free UAV. International Journal of Control, 84(11), 1873-1885.

Sikkel, L. N. C., Croon, G. C. H. E. d., Wagter, C. D., \& Chu, Q. P. (2016, October). A novel online model-based wind estimation approach for quadrotor micro air vehicles using low cost MEMS IMUs. In 2016 IEEE/RSJ International Conference on Intelligent Robots and Systems (IROS) (pp. 2141-2146).

Song, Y., Meng, Q. H., Luo, B., Zeng, M., Ma, S. G., \& Qi, P. F. (2016, December). A wind estimation method for quadrotors using inertial measurement units. In 2016 IEEE International Conference on Robotics and Biomimetics (ROBIO) (pp. 426-431).

Stotsky, A., \& Kolmanovsky, I. (2001). Simple unknown input estimation techniques for automotive applications. In American Control Conference (Vol. 5, pp. 3312-3317).

Tomić, T., \& Haddadin, S. (2014, September). A unified framework for external wrench estimation, interaction control and collision reflexes for flying robots. In 2014 IEEE/RSJ International Conference on Intelligent Robots and Systems (pp. 4197-4204).

Tomić, T., Schmid, K., Lutz, P., Mathers, A., \& Haddadin, S. (2016, October). The flying anemometer: Unified estimation of wind velocity from aerodynamic power and wrenches. In 2016 IEEE/RSJ International Conference on Intelligent Robots and Systems (IROS) (pp. 1637-1644).

Waslander, S., \& Wang, C. (2009, April). Wind Disturbance Estimation and Rejection for Quadrotor Position Control. In AIAA Infotech@Aerospace Conference. American Institute of Aeronautics and Astronautics.

Witte, B. M., Schlagenhauf, C., Mullen, J., Helvey, J. P., Thamann, M. A., \& Bailey, S. (2016, June). Fundamental Turbulence Measurement with Unmanned Aerial Vehicles (Invited). In 8th AIAA Atmospheric and Space Environments Conference. American Institute of Aeronautics and Astronautics.

Xiang, X., Wang, Z., Mo, Z., Chen, G., Pham, K., \& Blasch, E. (2016, September). Wind field estimation through autonomous quadcopter avionics. In 2016 IEEE/AIAA 35th Digital Avionics Systems Conference (DASC).

Xing, Z., Qu, Y., \& Zhang, Y. (2017, December). Shear wind estimation with quadrotor UAVs using Kalman filtering regressing method. In 2017 International Conference on Advanced Mechatronic Systems (ICAMechS) (pp. 196-201).

Yüksel, B., Secchi, C., Bülthoff, H. H., \& Franchi, A. (2014, July). A nonlinear force observer for quadrotors and application to physical interactive tasks. In 2014 IEEE/ASME International Conference on Advanced Intelligent Mechatronics (pp. 433-440).

Zhou, B. (2016, June). On asymptotic stability of linear time-varying systems. Automatica, 68, 266-276.

Zhou, B., \& Egorov, A. V. (2016, September). Razumikhin and Krasovskii stability theorems for time-varying time-delay systems. Automatica, 71, 281-291. 